\title{
Using theory of change in child health service interventions: a
}

\section{scoping review protocol [version 1; peer review: 2 approved]}

\author{
Benjamin Jones (Di)1, Shobhana Nagraj(iD1, Mike English (D)1,2 \\ ${ }^{1}$ Oxford Centre for Global Health Research, Nuffield Department of Medicine, University of Oxford, Oxford, UK \\ ${ }^{2}$ KEMRI-Wellcome Trust Research Programme, KEMRI-Wellcome Trust, Nairobi, Kenya
}

\author{
V1 First published: 28 Jan 2022, 7:30 \\ https://doi.org/10.12688/wellcomeopenres.17553.1 \\ Latest published: 28 Jan 2022, 7:30 \\ https://doi.org/10.12688/wellcomeopenres.17553.1
}

\section{Abstract}

Background: The objective of this scoping review is to map the evidence of how child health service interventions use their theory of change. A theory of change is a hypothesis of how and why an intervention is intended to bring about change. It can be used as a program design, implementation, and evaluation tool. This scoping review will provide an overview of the evidence base for, and identify the way in which, theories of change in child health service interventions are defined, rationalised, developed, presented, and refined.

Methods: The inclusion criteria for this scoping review is any child health service intervention globally, that describes their theory of change or theory of change development process. Relevant exclusions include: logic models or logic frameworks that do not meet this review's definition of theory of change, systematic reviews, behavioural change interventions that target patient's behaviour, school-based interventions, and maternal health interventions not related to child health outcomes. This scoping review will follow the Joanna Briggs Institute Reviewer's manual. Relevant publications will be first searched on selected electronic databases and grey literature. A search strategy will be developed. The search will be limited to articles written in the English language. Results of the search will be curated using Endnote and duplicates removed. Results will be imported to Rayyan. The inclusion criteria will be applied during the process of title and abstract screening, by two independent reviewers and disagreements resolved by a third independent reviewer. Fulltexts will have the inclusion criteria applied via the same reviewer process. Data relevant to the research sub-questions will be extracted, analysed, charted and discussed.

Ethics and dissemination: Ethical approval is not required for this review as we will make use of already published data. We aim to publish the findings of our review in a peer-reviewed journal.

\section{Open Peer Review}

\begin{tabular}{lcc} 
Approval Status & 1 \\
\hline & & 2 \\
version 1 & & \\
28 Jan 2022 & view & view
\end{tabular}

1. Solange Mianda (D), University of Western

Cape, Cape Town, South Africa

CHESAI, Cape Town, South Africa

2. Dale Barnhart ID, Harvard Medical School, Boston, USA

Any reports and responses or comments on the article can be found at the end of the article. 


\section{Keywords}

evaluation, intervention development, paediatrics, program theory, quality improvement, implementation science, theory of change

KEMRI wellcome

\section{This article is included in the KEMRI | Wellcome}

$$
\text { Trust gateway. }
$$

Corresponding author: Benjamin Jones (benjamin.jones@ndm.ox.ac.uk)

Author roles: Jones B: Conceptualization, Methodology, Resources, Software, Writing - Original Draft Preparation; Nagraj S: Conceptualization, Supervision, Writing - Review \& Editing; English M: Conceptualization, Supervision, Writing - Review \& Editing Competing interests: No competing interests were disclosed.

Grant information: This work was supported by Wellcome [207522; a Senior Fellowship award to ME] and the National Institute for Health Research [NIHR130812]. B] is also funded by the Nuffield Department of Medicine Prize Studentship as part of a University of Oxford DPhil program. This is supported by a Rhodes Scholarship funded by the Rhodes Trust. The funders had no role in study design, data collection and analysis, decision to publish, or preparation of the manuscript.

The funders had no role in study design, data collection and analysis, decision to publish, or preparation of the manuscript.

Copyright: (c) 2022 Jones B et al. This is an open access article distributed under the terms of the Creative Commons Attribution License, which permits unrestricted use, distribution, and reproduction in any medium, provided the original work is properly cited.

How to cite this article: Jones $B$, Nagraj $S$ and English M. Using theory of change in child health service interventions: a scoping review protocol [version 1; peer review: 2 approved] Wellcome Open Research 2022, 7:30 https://doi.org/10.12688/wellcomeopenres.17553.1

First published: 28 Jan 2022, 7:30 https://doi.org/10.12688/wellcomeopenres.17553.1 


\section{Introduction}

Health service interventions are interventions targeting the access to, and the use, costs, quality, delivery, organisation, financing, and outcomes of health care services such as hospitals and primary healthcare services (Institute of Medicine Committee on Health Services Research, 1994; Lohr \& Steinwachs, 2002; Steinwachs, 1991). An intervention is any organised activity, program, project, or initiative, that is supported by resources and established with the purpose of enacting change. This scoping review will focus on chid health service interventions, defined as any such intervention with a target population of children aged 0-19 years old. Health service interventions are often complex by nature, involving multiple stakeholders, diverse objectives, and are dependent in context. A theory of change (ToC) is a tool utilised by some health service interventions to navigate this complexity.

A theory of change (ToC) is a theory or hypothesis of how and why an intervention is intended to bring about change (Center for Theory of Change, 2021; Connell \& Kubisch, 1998; De Silva et al., 2014; Weiss, 1995). When a ToC is reported in internal or external organisation publications, it is an articulation of the hypothesised pathways that lead to change. It presents the cumulative study of an intervention's assumptions, activities, mechanisms, measurement indicators, outcomes, and context, as well as the linkages between these various components (Connell \& Kubisch, 1998).

A ToC can be presented in narrative form or illustrated visually using ToC diagrams. These diagrams are referred to by a number of different terms, depending on subtleties in their form and contents. These terms include, but are not limited to: logic models, logic frameworks, logframes, outcome hierarchies, and result chains. Inappropriate use of these terms can create confusion amongst the ToC literature (Weiss, 1997b). It is therefore important in this scoping review to explicitly outline what is meant and not meant by the term theory of change. For example, perhaps the most common term used incorrectly as a ToC synonym is logic model. De Silva et al. (2014) outline that logic models, strictly speaking, are more simple, linear, and rigid diagrams than true ToC diagrams, that do not outline assumptions, measurement indicators or describe a consideration of the intervention's causal relationships. 'Indicators' are the ToC terminology for the specific measurable marker used to determine if each activity performed in the proposed causal pathway and associated outcomes in a ToC, are achieved. This data can be either qualitative or quantitative. Indicators should include the population group related to the activity or outcome and the threshold that is the minimum accepted achievement of the outcome (Taplin \& Clark, 2012). An example a ToC diagram and a logic model have been included as Extended data to aid understanding (Jones, 2022).

The terms ToC, and the synonymous theory-based evaluation, are generally accepted to have been popularised in the 1990s, stemming from decades of work in program evaluation and program theory (Connell \& Kubisch, 1998; Connell et al., 1995; Weiss, 1995; Weiss, 1997b). They have been used in many fields, including health, education, business, community development and social welfare (Center for Theory of Change, 2021; De Silva et al., 2014; Weiss, 1997b). Despite this seemingly broad application base, the value that a ToC brings to an intervention needs to be considered if more organisations are to invest the resources required to articulate and present a comprehensive ToC. Even as early as 1987, HueyTsyh Chen and Peter Rossi argued for the internal and external validity of a theory based evaluation approach (Chen \& Rossi, 1987). Then in 1997, Carol Weiss, one of the architects behind the development of ToC articulation, noted that the literature was "replete with paeans to the value of the theory based approach" to program evaluation (Weiss, 1997a). That is, a number of studies speak to the benefits of the ToC development process such as helping to engage stakeholders, clarify focus, enhance connection to the intervention, and engage team thinking around the process that underpins interventions. Furthermore, these studies report the value that a ToC provides once completed, including providing a roadmap for the intervention, a clear anchor for evaluation, a base in which to refine thinking and monitor the intervention as context changes overtime, scale-up utility, and identify intentional and unintentional consequences (Anderson, 2006; Blamey \& Mackenzie, 2007; Connell \& Kubisch, 1998; De Silva et al., 2014; Rogers et al., 2004; Weiss, 1997a; Weiss, 1997b). However, it is important to consider that there is a selection bias of papers in the literature that present beneficial ToC articulations, rather than those that failed and were stopped short. Some issues with ToC identified in the literature include: the confusion around definition, that they are reductionist models of more open systems that give a false sense of control to the intervention implementers, they are not developed with enough rigour, and provide an excuse not to adapt when context changes (Connell \& Kubisch, 1998; De Silva et al., 2014; Weiss, 1995; Weiss, 1997a). Overall, this scoping review will aim to answer the question: how are ToCs for child health service interventions developed, utilised, and refined? It will also aim to answer the following research sub-questions:

1. How do these studies define ToC?

2. What is the rationale for the $\mathrm{ToC}$ being developed?

3. What is the process of development of the ToC?

4. Who is involved in the development of the ToC?

5. At what stage in the intervention are ToCs developed?

6. How are the ToCs presented in the literature?

7. In what way is the ToC used (purpose)?

8. Is the value of the ToC outlined, and if so what is it and the evidence supporting it?

9. How is the ToC refined overtime?

A preliminary search of MEDLINE, the Cochrane Database of Systematic Reviews and JBI Evidence Synthesis was conducted and no current or underway systematic reviews or scoping reviews on the topic were identified. Although no reviews were 
found, this initial search revealed a number of studies that may meet the eligibility criteria. For example, in 2016, Breuer et al. conducted a review of the use of ToC in public health interventions. This article acts as an example of a review of individual studies that describe how ToCs are developed and refined in healthcare. Another such paper, Kumar et al. (2020) uses a ToC to describe the service level interventions needed to be implemented to prevent retinopathy of prematurity and the associated indicators to monitor progress. Another, Makowiecka et al. (2019) established a common ToC for 61 unique maternal and newborn interventions, funded by a single philanthropic organisation. Finally, Hanson et al. (2019) developed a protocol of an evaluation study for a neonatal health project in India, which provides a detailed $\mathrm{ToC}$ and $\mathrm{ToC}$ development process attached as a specific appendix.

A scoping review was selected for this study as it will provide a mapping opportunity as well as an overview of the available research evidence; allowing for the identification of the way in which $\mathrm{ToC}$ in child health service interventions are defined, rationalised, developed, presented, and refined. This scoping review is intended to inform organisations working in the field of child health about how they may design and utilise their own ToC. Ultimately, the objective of this scoping review is to map the evidence how child health service interventions around the world develop, use, and refine their theory of change.

\section{Methods}

The proposed scoping review will be conducted in accordance with the JBI methodology for scoping reviews (Joanna Briggs Institute, 2015; Peters et al., 2020) and was also used for the development of this protocol.

\section{Eligibility criteria}

Participants. Health service interventions targeted at children and youth aged 0-19 years will be included. Health service interventions will be defined as interventions targeting the access to, and the use, costs, quality, delivery, organisation, financing, and outcomes of health care services. The term 'intervention' will be defined as any organised activity, program, project, or initiative, that is supported by resources and established with the purpose of enacting change. This scoping review will exclude studies in which the main target of the intervention was individual patients rather than the health service more broadly. For example, an intervention using behavioural change theory aiming to influence a patient's cognition and behaviour would be excluded. However, if an intervention used behavioural change theory aiming to change the behaviour of staff members in order to more effectively deliver a healthcare service, this will be included. Settings outside healthcare facilities such as schools, will be excluded. Obstetric health service interventions that aim to improve maternal health outcomes rather than newborn health outcomes will also be excluded. Some health service interventions that overlap with public health interventions such as vaccination programs will be included in this scoping review.
Concept. Interventions that discuss their ToC will be included. Specifically, papers will be included if they a) describe how a ToC was utilised throughout any stage of a child health service intervention such as design, implementation, or evaluation. Or b) describe the development process for a $\mathrm{ToC}$ planned to be used in a child health service intervention. A ToC will be defined as a theory or hypothesis of how and why an intervention is intended to bring about change. As highlighted by Breuer et al. (2016), it may be difficult to ascertain if a study has discussed a ToC as per this definition purely from the title and abstract alone. Therefore, during title and abstract screening a study will be included for full-text screening if it refers to the development of their own ToC, theory-based evaluation, theory informed evaluation, program theory, outcomes hierarchy, results chain logic model, or logic framework or, if the title/abstract include comments on the steps of how, or mechanisms behind why, an intervention worked. Then, during full-text screening, when a more thorough analysis of how these studies used the various terms above can be conducted, the specific definition of ToC above will be applied. The included studies should include a consideration of at least one of the following ToC components; assumptions, activities, mechanisms, measurement indicators, outcomes, and context, or the linkages between these various components.

Sources. This scoping review will consider a number of different study designs including both quantitative, qualitative and mixed-methods study designs. From the grey literature, organisational e.g., private organisations and NGO's as well as government ToC documents will be included. Systematic reviews will not be included. There will be no other limits of date, study design, or type of publication.

\section{Search strategy}

The search strategy will aim to locate both published and unpublished studies. An initial limited search of MEDLINE was conducted to identify articles on the topic. The text words contained in the titles and abstracts of relevant articles, and the index terms used to describe the articles were used to develop full search strategies for each database (see Extended data). The search strategy, including all identified keywords and index terms, will be adapted for each included database and/or information source. Broadly, the database search will combine terms from the two themes: childhood age range and ToC. The reference list of all included sources of evidence will be screened for additional studies.

Studies published in English will be included. This is due to resourcing constraints within the research team. No publication year restrictions will be used in the search in order to gain an understanding of how different terminology has been used over time.

The databases to be searched include, MEDLINE, EMBASE, Global Health, WHO Global Index Medicus, CINAHL and SCOPUS. Sources of unpublished studies/ grey literature to be searched include the first 10 pages of a specific google 
search 'Child* "theory of change" filetype:pdf'. The titles and then full-texts of these pdfs will then have the same criteria applied as the literature. The most recent search of these sources in formulating the search strategy was completed on $14^{\text {th }}$ December 2021.

\section{Evidence selection}

This will be an iterative process whereby the literature will be searched and the search strategy refined. Following the search, all identified citations will be collated and uploaded into Endnote V20 and duplicates removed before being uploaded to Rayyan, an online review management software. Following a pilot test, titles and abstracts will then be screened by two or more independent reviewers for assessment against the inclusion criteria for the review. The inclusion and exclusion criteria were refined and agreed by all three researchers. As outlined in the introduction, there are a number of terms used to describe diagrams that are similar to, but often lack the depth of, a complete ToC. This presents a challenge during particularly the title and abstract screening stage, where a paper may not explicitly acknowledge an articulation of a ToC and label it as such. Therefore, careful analysis of the abstracts to identify if comments are made on the 'how' and 'why' an intervention has worked, will be critical when there is an absence of specific phrases such as; ToC, theory based evaluation, theory informed evaluation, logic model, logic framework, or program theory. This approach to study selection diverges from that of the review conducted by Breuer et al. (2016) who limited the criteria to needing the specific term 'theory of change' to be explicitly mentioned in the title or abstract order for the paper to meet the inclusion criteria.

Following this, potentially relevant sources will be retrieved in full. The full text of selected citations will be assessed in detail against the inclusion criteria by two or more independent reviewers. Reasons for exclusion of sources of evidence at full text that do not meet the inclusion criteria will be recorded and reported in the scoping review. Any disagreements that arise between the reviewers at each stage of the selection process will be resolved through discussion. The results of the search and the study inclusion process will be reported in full, in the final scoping review and presented in a Preferred Reporting Items for Systematic Reviews and Meta-analyses extension for scoping review (PRISMA-ScR) flow diagram.

\section{Data collection}

Data will be extracted from papers included in the scoping review by two or more independent reviewers using a data extraction tool developed by all reviewers, to aid with consistency of which data will be extracted (see Extended data). The data extracted will include specific details about the participants, concept, context, study methods and key findings relevant to the review questions. A draft extraction form is provided (see Extended data). The draft data extraction tool will be modified and revised as necessary during the process of extracting data from each included evidence source. Modifications will be detailed in the scoping review. Any disagreements that arise between the reviewers will be resolved through discussion, or with an additional reviewer(s). If appropriate, authors of papers will be contacted to request missing or additional data, where required.

Data analysis and presentation. Data will be presented in table form. Furthermore, a narrative summary will accompany the tabulated information and will describe how the results relate. Data will be analysed using both qualitative and quantitative methods. Qualitative data analysis will involve all reviewers engaging in an in-depth discussion about the ToC data and describing the major concepts arising. Literature will be analysed to study location, type of health service intervention, and $\mathrm{ToC}$ definition (using a checklist of key ToC components such as assumptions, activities, mechanisms, measurement indicators, outcomes, context, and linkages), ToC presentation. Data extracted from the data extraction tool (see Extended data) will then be charted as per the JBI. Firstly, in the data charting process, a calibration exercise with the full team will be implemented. This will involve a random sample of 20 citations for title and abstract screening. A roundtable discussion will be had to clarify any issues. After the initial calibration exercise, one author (BJ) will chart the data independently and two authors (ME and $\mathrm{SN}$ ) will verify the data for accuracy. Inconsistencies and disagreements will be resolved through discussion. An iterative approach will be taken with the data charting process and major revisions with rationale will be included in the final report. Critical appraisal of individual sources of evidence will not be completed as it is out of the scope of this review.

This scoping review has a number of potential impacts. Firstly, it will further elucidate to implementation researchers how $\mathrm{ToC}$ are described in the literature, what is meant by the term $\mathrm{ToC}$, the value that ToCs bring to interventions, what is missing from these descriptions, and encourage consideration of how these descriptions could be improved. Secondly, for health service practitioners working within the field of child health service interventions, it will map the available evidence on how these interventions may use a ToC. Thirdly, this scoping review may encourage those in fields outside of child health service interventions to consider using a $\mathrm{ToC}$ as a program design, implementation, and evaluation tool.

Study status

This scoping review will be conducted in early 2022 .

\section{Data availability}

Underlying data

No underlying data are associated with this article.

\section{Extended data}

Open Science Framework: Using theory of change in child health service interventions: a scoping review protocol. (Jones, 2022)

https://doi.org/10.17605/OSF.IO/5TPGM

This project contains the following extended data:

- $\quad$ Search strategies.docx 
- Data extraction instrument.docx

- Theory of change diagram vs logic model examples. docx
Data are available under the terms of the Creative Commons Zero "No rights reserved" data waiver (CC0 1.0 Public domain dedication).
Anderson AA: The Community Builder's Approach to Theory of Change: A Practical Guide to Theory Development. Aspen Institute Roundtable on Community Change. 2006 Reference Source

Blamey A, Mackenzie M: Theories of Change and Realistic Evaluation: Peas in a Pod or Apples and Oranges? Evaluation. 2007; 13(4): 439-455. Publisher Full Text

Breuer $\mathrm{E}$, Lee $\mathrm{L}$, De Silva $\mathrm{M}$, et al.: Using theory of change to design and evaluate public health interventions: a systematic review. Implement Sci. 2016; 11: 63.

PubMed Abstract | Publisher Full Text | Free Full Text

Center for Theory of Change: What is Theory of Change? 2021.

Reference Source

Chen HT, Rossi PH: The Theory-Driven Approach to Validity. Evaluation and Program Planning. 1987; 10(1): 95-103.

Publisher Full Text

Connell J, Kubisch A: Applying a Theory of Change Approach to the Evaluation of Comprehensive Community Initiatives: Progress, Prospects, and Problems. In New Approaches to Evaluat-ing Community Initiatives, Theory, Measurement, and Analysis. Washington DC: Aspen Institute. 1998; 2 Reference Source

Connell J, Kubisch A, Schorr L, et al:: New Approaches to Evaluating Community Initiatives: Concepts, Methods and Contexts. New York: Aspen Institute. 1995.

Reference Source

De Silva MJ, Breuer E, Lee L, et al.: Theory of Change: a theory-driven approach to enhance the Medical Research Council's framework for complex interventions. Trials. 2014; 15(1): 267.

PubMed Abstract | Publisher Full Text | Free Full Text

Hanson C, Zamboni K, Prabhakar V, et al.: Evaluation of the Safe Care, Saving Lives (SCSL) quality improvement collaborative for neonatal health in Telangana and Andhra Pradesh, India: a study protocol. Glob Health Action. 2019; 12(1): 1581466

PubMed Abstract | Publisher Full Text | Free Full Text

Institute of Medicine Committee on Health Services Research: A Working Definition of Health Services Research. 1994.

Reference Source

Joanna Briggs Institute: Joanna Briggs Institute Reviewers' Manual: 2015.
edition/Supplement. Retrieved from Australia: 2015.

Reference Source

Jones B: Using theory of change in child health service interventions: a scoping review protocol. 2022.

http://wwww.doi.org/10.17605/OSF.IO/5TPGM

Kumar P, Chawla D, Thukral A, et al.: Development of a quality improvement package for reducing sight-threatening retinopathy of prematurity. Indian J Ophthalmol. 2020; 68(Suppl 1): S115-S120.

PubMed Abstract | Publisher Full Text | Free Full Text

Lohr KN, Steinwachs DM: Health services research: An evolving definition of the field. Health Serv Res. 2002; 37(1): 7-9.

PubMed Abstract

Makowiecka K, Marchant T, Betemariam W, et al:: Characterising innovations in maternal and newborn health based on a common theory of change: lessons from developing and applying a characterisation framework in Nigeria, Ethiopia and India. BMJ Glob Health. 2019; 4(4): e001405. PubMed Abstract | Publisher Full Text | Free Full Text

Peters MDJ, Marnie C, Tricco AC, et al.: Updated methodological guidance for the conduct of scoping reviews. JBI Evid Synth. 2020; 18(10): 2119-2126. PubMed Abstract | Publisher Full Text

Rogers P, Petrosino, A, Huebner T, et al.: Program Theory Evaluation: Practice, Promise, and Problems. New Directions for Evaluation. 2004; 2000: 5-13. Reference Source

Steinwachs D: Health-Services Research - Its Scope and Significance. American Journal of Pharmaceutical Education. 1991; 55(3): 274-278.

Taplin D, Clark H: Theory of Change Basics A Primer on Theory of Change. Retrieved from New York: 2012

Reference Source

Weiss $\mathrm{CH}$ : Nothing as Practical as Good Theory: Exploring Theory-Based Evaluation for Comprehensive Community-Based Initiatives for Children and Families. In New Approaches to Evaluating Community Initiatives, Concepts, Methods and Contexts. Washington, DC: Aspen Institute. 1995; 1. Reference Source

Weiss $\mathrm{CH}$ : How can theory-based evaluation make greater headway? Evaluation Review. 1997a; 21(4): 501-524.

Publisher Full Text

Weiss CH: Theory-based evaluation: Past, present, and future. New Dir Eval. 1997b; 1997(76): 41-55.

Publisher Full Text 


\section{Open Peer Review}

\section{Current Peer Review Status:}

\section{Version 1}

Reviewer Report 25 February 2022

https://doi.org/10.21956/wellcomeopenres.19410.r48374

(C) 2022 Barnhart D. This is an open access peer review report distributed under the terms of the Creative Commons Attribution License, which permits unrestricted use, distribution, and reproduction in any medium, provided the original work is properly cited.

\section{Dale Barnhart}

Department of Global Health and Social Medicine, Harvard Medical School, Boston, MA, USA

This study protocol describes the process through which the authors will conduct a scoping review of the use of theories of change in child health service interventions. The author's goals of understanding how theories of changes are developed and used in this context are well defined. The search strategy is well-defined, and the research team has thoughtfully considered how to account for mislabeled "theories of change" by proposing a clear definition for the term and including related terms in the search criteria. Data collection tools are provided; however, as this is a protocol for a future study, the data itself is not yet available.

Is the rationale for, and objectives of, the study clearly described?

Yes

Is the study design appropriate for the research question? Yes

Are sufficient details of the methods provided to allow replication by others? Yes

Are the datasets clearly presented in a useable and accessible format? Not applicable

Competing Interests: No competing interests were disclosed.

Reviewer Expertise: implementation science; epidemiology

I confirm that I have read this submission and believe that I have an appropriate level of expertise to confirm that it is of an acceptable scientific standard. 
Reviewer Report 18 February 2022

https://doi.org/10.21956/wellcomeopenres.19410.r48376

(C) 2022 Mianda S. This is an open access peer review report distributed under the terms of the Creative Commons Attribution License, which permits unrestricted use, distribution, and reproduction in any medium, provided the original work is properly cited.

\section{Solange Mianda}

1 School of Public Health, University of Western Cape, Cape Town, South Africa

2 CHESAI, Cape Town, South Africa

Thank you for the opportunity to review this paper I have read the paper with interest and I think the background idea is interesting. Child health is a growing global concern, and implementing and/or evaluating health service programmes with the promise to improve child health become imperative. While thinking of programmes that may provide better gains for child survival, developing a good theory of change (TOC) in the implementation and/or evaluation of such programmes could bear multiple benefits as enumerated by the authors. Hence, the need to better understand how TOC is defined (clarity of concept), developed and used (the object of this review protocol).

\section{Comments to the authors:}

I have found that the rationale for, and the study were clearly described. I would like to subject that the heading 'objective' be included in the paper to guide the reader on the flow of the steps followed in the review. As it stands, the objective seems lost in the introduction.

The sentence "This scoping review is intended to inform organisations working in the field of child health about how they may design and utilise their own ToC", seems restrictive. There may be individuals e.g. students, public health practitioners, health promoters who may be interested in designing their own TOC for particular interventions and not be part of a given organization.

If "this scoping review may encourage those in fields outside of child health service interventions to consider using a ToC as a program design, implementation, and evaluation tool" as stated by the authors on the potential impacts of this review, then the statement in the above paragraph should be corrected to expand to include a broader audience. In this sentence, I wonder whether the development, refining and use of TOC is done by researchers (designers of interventions, or interventions) "Ultimately, the objective of this scoping review is to map the evidence of?? how child health service interventions around the world develop, use, and refine their theory of change"

The authors opted for the JBI methodology for scoping reviews (Joanna Briggs Institute, 2015; Peters et al., 2020) although appropriate for the scope of study proposed by the authors, I did not find the rationale for this choice, given the existence of other approaches to scoping reviews. The authors have sufficiently provided details of the methods to allow replication by others.

\section{Participant}


The authors have stated that Health service interventions targeted at children and youth aged 0-19 years will be included in the review. What is the rationale for the choice of this age range? What is the definition of "child" in this review? The title is restricted to child, but the inclusion criteria is extended to youth. Providing a clear definition of who the authors consider as "child" needs to be provided to avoid confusion.

'Health service interventions' is a broad term and could be restrictive, Have the authors thought of interventions using terms as quality improvement or implementation? Many interventions make use of these terms and will not necessarily use intervention in the title, how would the authors account for articles that could be missed should these terms not be included in the search terms?

I look forward to seeing the end product, sounds very interesting, best wishes to the authors

Is the rationale for, and objectives of, the study clearly described?

Yes

Is the study design appropriate for the research question?

Yes

Are sufficient details of the methods provided to allow replication by others?

Yes

Are the datasets clearly presented in a useable and accessible format?

Yes

Competing Interests: No competing interests were disclosed.

Reviewer Expertise: Health systems governance, health policy and systems research, maternal and child health, implementation research, health systems strengthening, programme evaluation, evidence synthesis

I confirm that I have read this submission and believe that I have an appropriate level of expertise to confirm that it is of an acceptable scientific standard. 